\title{
Epidermal and Dermal Hallmarks of Photoaging are Prevented by Treatment with Night Serum Containing Melatonin, Bakuchiol, and Ascorbyl Tetraisopalmitate: In Vitro and Ex Vivo Studies
}

Mridvika Narda • Anthony Brown • Béatrice Muscatelli-Groux •

Jean A. Grimaud · Corinne Granger

Received: November 18, 2019 / Published online: January 3, 2020

(C) The Author(s) 2020

\begin{abstract}
Introduction: Photoaging is a complex process that is chiefly the result of oxidative stress caused by ultraviolet (UV)-generated reactive oxygen species. To counter this process, we developed a 3-in-1 night facial serum (3-in-1 NFS) containing a combination of direct and indirect antioxidants and polyphenols that is designed to attenuate UV-generated free radicals and stimulate dermal protein synthesis. In clinical trials 3-in-1 NFS improved the appearance of photoaged skin. In this study we sought to identify some of the main histologic changes responsible for this.
\end{abstract}

Enhanced Digital Features To view enhanced digital features for this article go to https://doi.org/10.6084/ m9.figshare.11371665.

M. Narda $(\bowtie)$ C C. Granger

Innovation and Development, ISDIN, Barcelona, Spain

e-mail: mridvika.narda@isdin.com

A. Brown

External Consultant to ISDIN, Barcelona, Spain

B. Muscatelli-Groux · J. A. Grimaud

MATRISCIENCE SAS, Paris Santé Cochin, Paris,

France
Methods: We performed an immunolabeling analysis of some of the salient epidermal and dermal proteins in 3-in-1 NFS-treated primary epidermal keratinocytes (HEKs) and dermal fibroblasts (HDFs) in vitro, and in UV-exposed skin explants ex vivo. Numbers of apoptotic sunburn cells following exposure of 3-in-1 NFStreated skin explants to UV radiation were also determined.

Results: We demonstrate that 3-in-1 NFS increases levels of filaggrin and aquaporin 3 in HEKs, and levels of collagen I and collagen III in HDFs in vitro. Levels of precursor procollagen type I and tropoelastin were increased in ex vivo skin explants. Numbers of apoptotic sunburn cells were significantly reduced in UV-exposed skin explants. These effects were only observed with the combination of ingredients in 3-in-1 NFS, suggesting that they have a synergistic effect on photoaged skin biology.

Conclusion: Our results show that some of the histological hallmarks of photoaging are improved with the use of 3-in-1 NFS.

Keywords: Antioxidant; Ascorbyl tetraisopalmitate; Bakuchiol; Melatonin; Polyphenol; Photoaging; Skin aging; Ultraviolet 


\section{Key Summary Points}

\section{Why carry out this study?}

Facial photoaging significantly impacts quality of life and self-esteem. Effective and well-tolerated anti-photoaging treatments are therefore needed.

To identify some of the main histologic changes responsible for the clinical improvement seen in photoaged skin following 3-in-1 NFS treatment.

\section{What was learned from the study?}

Use of 3-in-1 NFS increases the expression of proteins that restore the skin's barrier function, hydration levels, and help reverse dermal protein atrophy.

The components of 3-in-1 NFS synergize to uniquely protect the skin from the effects of UV radiation.

\section{INTRODUCTION}

Skin serves as interface between the body's internal structures and its external environment, and is the site upon which the cumulative effects of age and its external environment visibly converge. These extrinsic factors cause gross morphological and physiological changes in skin that superimpose upon the natural aging process. The most important environmental factor leading to extrinsically aged skin is solar ultraviolet radiation (UVR). Chronic exposure of skin to solar UVR leads to a premature aging phenotype characterized by deep wrinkles, laxity, and a leathery appearance known as photoaging [1].

Photoaged skin is characterized by massive loss of dermal collagen that is the net result of reduced type I and III procollagen synthesis [2] and increased degradation of mature collagen by metalloproteinases (MMPs) [3]. This results in irregular distribution of collagen within the dermis with areas completely devoid of collagen fibers adjacent to dense clumps of collagenous material [4]. UVR exposure also reduces the synthesis of elastin and increases elastic fiber degradation [5]. Additionally, disorganized and non-functional elastic fibers accumulate within the dermis, making photoaged skin appear yellow and leathery [6]. Together the breakdown of the normal collagen and elastin reduces skin's elasticity and tensile strength, resulting in sagging and wrinkling $[7,8]$. Chronic sun exposure also perturbs the structural integrity of the top layer of the epidermis, the stratum corneum (SC), and alters its hydration and lipid properties, as well as its thickness, color, and lightabsorbing properties [9-11].

Crucially both the epidermis and dermis are capable of self-repair and this natural process can be augmented by application of exogenous substances that stimulate the skin's homeostatic responses. To date, only retinoids have been shown to unequivocally improve the appearance of photoaged skin [12]. Their use, however, is compromised by undesirable side effects such as pruritus, burning, erythema, peeling, and photosensitivity [12]. To address this, we developed a serum-in-oil night facial serum (3in-1 NFS) that incorporates three ingredients which actively support the biological processes compromised in photoaged skin. Melatonin (5acetyl-5-methoxytryptamine) is a pineal hormone that acts as both an indirect antioxidant, by inducing antioxidative gene expression, and a free radical scavenger [13, 14]. Bakuchiol (4[(1E,3S)-3-ethenyl-3,7-dimethyl-1,6-octadien-1yl]phenol) is a naturally occurring phenolic compound with retinol-like properties [15]. Bakuchiol induces collagen expression in cultured fibroblasts and activates the expression of genes involved in antioxidant defense $[15,16]$. Bakuchiol is also thought to act as a direct free radical scavenger [17]. Clinically, bakuchiol was shown to improve signs of photoaging, including wrinkling and hyperpigmentation, to a degree comparable to retinol [18]. Ascorbyl tetraisopalmitate (ATIP) is a lipophilic and nonoxidizable form of vitamin C [19]. As well as possessing antioxidant and anti-inflammatory properties, ATIP also increases the skin's hydration and smoothness $[19,20]$. Clinically these three components resulted in skin that 
was firmer, less wrinkled, and more hydrated in subjects after nightly application of the serum for 3 months [21]. In order to identify some of the histological changes likely responsible for this, we performed an immunocytochemical analysis of primary human keratinocytes and fibroblasts cultured in the presence of different concentrations of 3-in-1 NFS, and an immunohistochemical analysis of ultraviolet (UV)-exposed skin explants topically treated with 3-in-1 NFS and each of its constituents in isolation.

\section{METHODS}

\section{In Vitro Study}

\section{Cell Culture and Immunocytochemistry}

Primary adult human epithelial keratinocytes (HEKs) and primary human dermal face fibroblasts (HDFs) were incubated with $500 \mu \mathrm{L} /$ well of 3 -in-1 NFS-containing media $(0.05 \%, 0.1 \%$, $0.5 \%$, and $1 \%$ ) for $24 \mathrm{~h}$ at $37{ }^{\circ} \mathrm{C}$. Following 3-in1 NFS treatment, cells were fixed and stained with the following primary antibodies: rabbit anti-collagen I (\#20111, Novotec, Bron, France), rabbit anti-collagen III (\#20311, Novotec, Bron, France), rabbit anti-laminin (\#24811, Novotec, Bron, France), rabbit anti-fibronectin (\#24911, Novotec, Bron, France), rabbit anti-aquaporin 3 (\#ab153694, Abcam, Cambridge, UK), and rabbit anti-filaggrin (\#PAJ103Hu01, Cloud-Clone Corp, Katy, TX) overnight at $37^{\circ} \mathrm{C}$. Cells were washed and then incubated with secondary antibody (Alexa Fluor 488 goat anti-rabbit IgG [\#A-11008, Thermo Fisher Scientific, Waltham, $\mathrm{MA}]$ ) at room temperature for $2 \mathrm{~h}$. Finally, cells were incubated with DAPI (for nucleic acid staining) at room temperature for $10 \mathrm{~min}$. Five independent experiments were performed for each condition.

\section{Image Acquisition and Analysis}

Fluorescence images were obtained using a Nikon Ti-S microscope (Nikon, Tokyo, Japan) and the fluorescent signal was quantified using MetaMorph $^{\circledR}$ software (Molecular Devices, San Jose, CA, USA). For collagen I/III, laminin, and fibronectin, values were calculated as fluorescence intensity of the protein/nucleus number.
For aquaporin 3 (AQP3) and filaggrin (FLG) it was calculated as fluorescence intensity of the protein/fluorescence intensity of DAPI (nucleus). Differences between untreated control and treated cells were determined by means of a two-tailed Student's $t$ test. A $p$ value less than 0.05 was considered significant.

\section{Ex Vivo Study}

\section{Antiaging Treatment}

Abdominal skin explants (NativeSkin ${ }^{\circledR}$, Genoskin, Toulouse, France) were generated from two healthy Caucasian women (30 and 32 years of age, Fitzpatrick type II) undergoing abdominoplasty. Two explants per donor were used for subsequent studies.

Anti-photoaging effects of the following formulations were assessed: (1) 3-in-1 NFS, (2) vehicle, (3) ATIP, (4) melatonin, (5) bakuchiol, and (6) the combination of melatonin and bakuchiol (Mel/Bak). ATIP, melatonin, bakuchiol, and Mel/Bak were used at the same concentrations as those in 3-in-1 NFS and in the same vehicle (see Table 1 for full vehicle composition). Briefly, $10 \mu \mathrm{L}$ of each test item was evenly applied to the surface of NativeSkin ${ }^{\circledR}$ units $1 \mathrm{~h}$ after $\mathrm{UV}$ radiation $\left(12.5 \mathrm{~J} / \mathrm{cm}^{2} \mathrm{UVA}\right.$ and $50 \mathrm{~mJ} / \mathrm{cm}^{2} \mathrm{UVB}$ ) for 4 consecutive days. A silicone ring was firmly fixed onto the biopsy to prevent topically applied formulations from leaking into the culture medium. Before each application, a cotton swab was used to remove the previous day's treatment and the surface of the explant was washed twice with PBS. Following the fourth UV treatment cycle, formulations were left on the surface of the skin for $2 \mathrm{~h}$ prior to fixation and paraffin embedding.

Table 1 Vehicle composition

\section{Composition}

Vehicle Caprylic/capric triglyceride, dicaprylyl carbonate, squalane, alcohol denat., caprylyl glycol, 1,2hexanediol, PEG-8; aqua, tocopherol, ascorbyl palmitate, citric acid, ascorbic acid 


\section{Quantification of Sunburn Cells}

Five-micron skin sections were stained with hematoxylin and eosin (H\&E). Representative images were then captured using a NanoZoomer S360 Digital Slide Scanner (Hamamatsu Photonics, Hamamatsu, Japan). The number of sunburn cells (SBCs) (keratinocytes with pyknotic nuclei) in each section was visually determined. A total of 40 images were analyzed per experimental condition (10 images per replicate; 2 replicates per donor; 2 donors per experimental condition). For each replicate, donor and experimental condition, the mean \pm standard error (SEM) was calculated. Data was analyzed by Dunnett's multiple comparisons test. A $p$ value less than 0.05 was considered significant.

\section{Immunohistochemistry}

Five-micron skin sections were stained with rat anti-procollagen type I (\#ab64409, Abcam, Cambridge, UK) and rabbit anti-tropoelastin (\#ab21600, Abcam, Cambridge, UK) antibodies overnight at $4{ }^{\circ} \mathrm{C}$. Slides were washed and then incubated with secondary antibodies (goat antirat Alexa 546 [\#A-11081, Invitrogen, Carlsbad, CA] or goat anti-rabbit Alexa 488 [\#ab150077, Abcam, Cambridge, UK]) for $1 \mathrm{~h}$ at room temperature and counterstaining of cell nuclei was performed using DAPI. For all experimental conditions, a control incubated without primary antibodies was included.

\section{Image Acquisition and Analysis}

Immunostained slides were mounted with antifading medium (Fluoromount ${ }^{\mathrm{TM}}$, Sigma Aldrich, St. Louis, MO) and imaged with a Nanozoomer S360 Digital Slide Scanner (Hamamatsu Photonics, Hamamatsu, Japan) and NDP.view2 software (Hamamatsu Photonics, Hamamatsu, Japan). Procollagen type I and tropoelastin expression was determined by calculating the percentage area of the dermis using Image J software (NIH, Bethesda, MD). A total of 64 images per experimental condition were analyzed ( 2 donors per experimental condition;
2 skin explants per donor; 2 tissue sections per explant; 8 images per tissue section). Statistical analysis was performed considering the mean values of both donors using a Games-Howell post hoc test to compare between conditions. A $p$ value less than 0.05 was considered significant.

\section{Ethical Approval and Informed Consent}

All human skin explants used in this study were obtained from abdominal surgical residues after written informed consent from the donors and in full respect of the Declaration of Helsinki and article L.1245 of the French Public Health Code [22]. The latter does not require any prior authorization by an ethics committee for use of surgical waste.

\section{RESULTS}

\section{In Vitro Study}

\section{Effect of 3-in-1 NFS on FLG and AQP3 Expression}

In clinical trials, 3-in-1 NFS was shown to reduce transepidermal water loss (TEWL) and increase hydration levels [21]. We therefore suspected that 3-in-1 NFS may influence the expression of key molecules involved in skin barrier formation and epidermal water transport, such as FLG [23] and AQP3 [24]. Expression levels of FLG and AQP3 were examined in primary adult HEKs cultured in media supplemented with $0.05 \%, 0.1 \%, 0.5 \%$, and $1 \%$-in-1 NFS. FLG expression was significantly increased in the presence of 3-in-1 NFS at all concentrations tested, with the greatest effects observed at $0.05 \%$ and $0.1 \%$ (Fig. 1a). A dose-dependent increase in AQP3 expression was observed (Fig. 1b).

\section{Effect of 3-in-1 NFS on Dermal Proteins}

Clinically, 3-in-1 NFS reduced facial wrinkles [21], suggesting it may influence dermal protein levels in photoaged skin. We therefore examined the effect of 3-in-1 NFS on the expression of dermal proteins by culturing primary HDFs in 
A

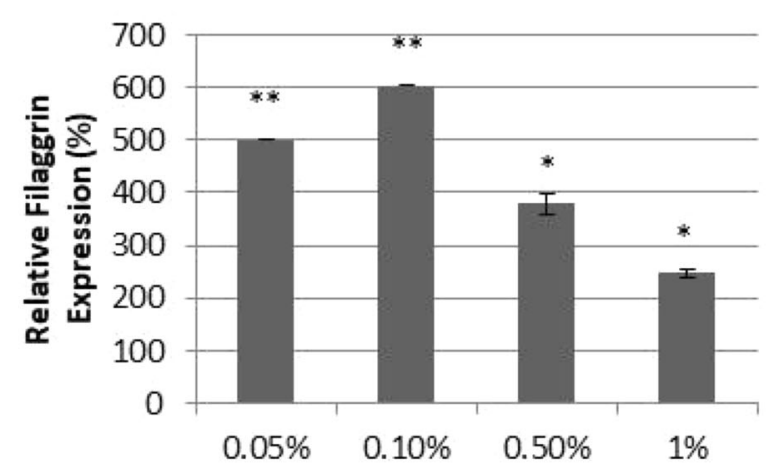

C

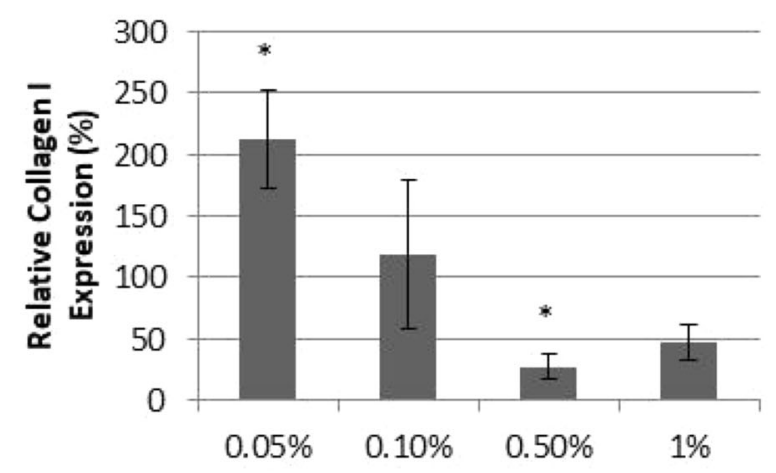

E

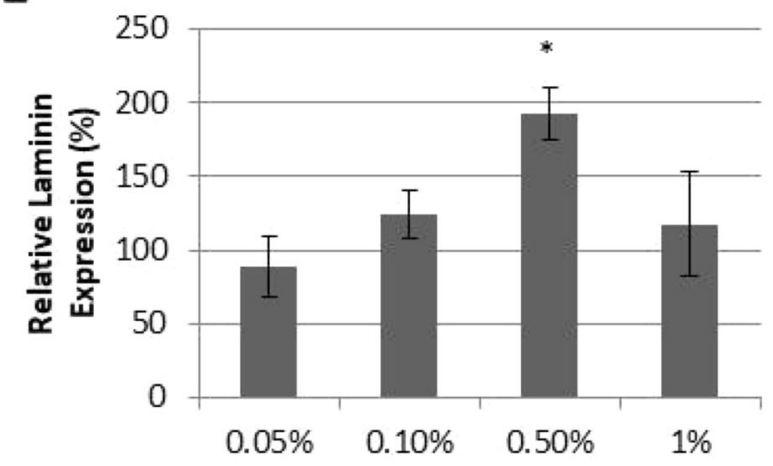

Fig. 1 Effect of 3-in-1 NFS on keratinocytes and fibroblasts. a FLG expression, b AQP3 expression, c collagen I expression, $\mathbf{d}$ collagen III expression, e laminin expression, and $\mathbf{f}$ fibronectin expression in fibroblasts treated with

the presence of 3-in-1 NFS. Expression of collagen I and III, the most abundant dermal proteins, was higher in HDFs treated with $0.05 \%$
B

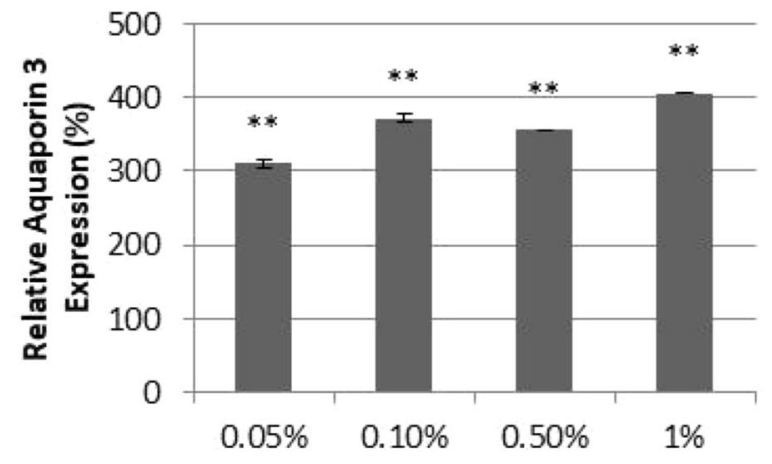

D

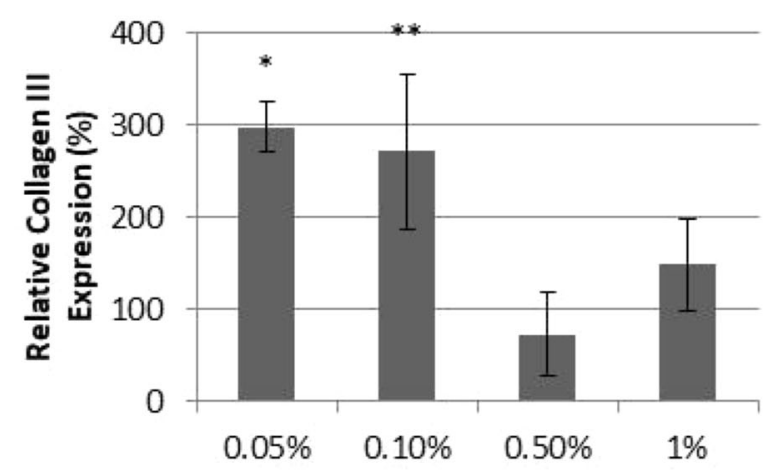

F

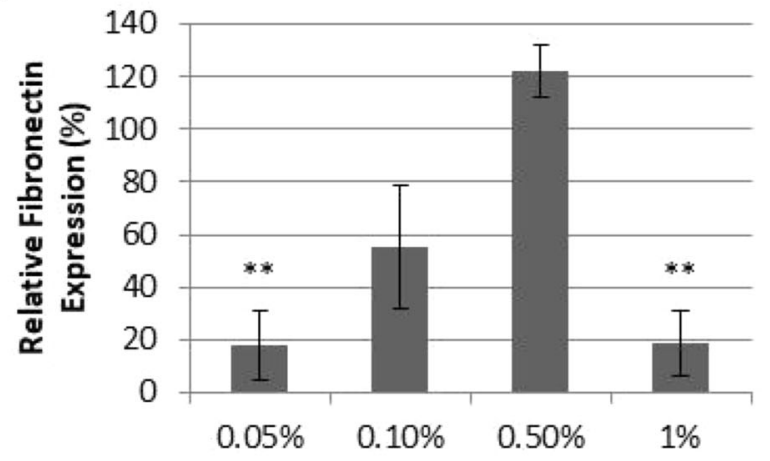

3-in-1 NFS (0.05\%, 0.1\%, 0.5\%, 1\%). Graphs show mean protein expression levels $( \pm$ SEM) of 5 independent experiments relative to those of untreated control cells (100\%). ${ }^{*} p<0.05 ;{ }^{* *} p<0.01$

and $0.1 \%$ 3-in-1 NFS, but was inhibited at higher concentrations (Fig. 1c, d). Laminin, a major component of basement membranes that 
separates the epidermis from the dermis [25], was only increased at $0.5 \%$ (Fig. 1e). Fibronectin, a large multicomponent glycoprotein that plays an important role in the organization and maintenance of the extracellular matrix (ECM) [26], was inhibited at both low $(0.05 \%$ and $0.1 \%$ ) and high concentrations $(1 \%)$ of 3 -in-1 NFS. Only 0.5\% 3-in-1 NFS had no impact upon fibronectin expression (Fig. 1f).

\section{Ex Vivo Study}

\section{Effect of 3-in-1 NFS on Sunburn Cell Formation}

Explants treated with 3-in-1 NFS presented with significantly fewer SBCs following UV exposure than untreated skin explants $(4.75 \mathrm{SBCs} /$ donor vs. $8.5 \mathrm{SBCs} /$ donor, respectively; $p<0.05$ ). Indeed, numbers of SBCs in 3-in-1 NFS-treated skin were similar to those in non-irradiated skin (3.5 SBCs/donor; $p=$ not significant [n.s.]). Conversely, the number of SBCs in vehicletreated skin (9.75 SBC/donor; $p=$ n.s.), and in skin treated with the individual components of 3-in-1 NFS alone (melatonin 9.25 SBC/donor; bakuchiol $10 \mathrm{SBC} /$ donor; ATIP $9 \mathrm{SBC} /$ donor; $p=$ n.s., all), or the combination of melatonin and bakuchiol (9.25 SBC/donor; $p=$ n.s.) were similar to those of untreated UV-exposed skin (Fig. 2).

\section{Effect of 3-in-1 NFS on Collagen Biosynthesis}

Our in vitro study suggested that 3-in-1 NFS may help restore collagen deficit in photoaged skin by stimulating new collagen synthesis in dermal fibroblasts. To determine whether this effect was also observed in photoaged skin when topically applied, we sought to examine the effect of 3-in-1 NFS on the precursor of collagen I, procollagen type I, in skin explants chronically exposed to UVR.

Treatment with 3-in-1 NFS increased the expression of procollagen type I in irradiated skin explants by $78.8 \%(p<0.01)$ relative to untreated irradiated skin explants (Fig. 3a, b). Interestingly, neither the individual components of 3-in-1 NFS (ATIP $+1.88 \%$; bakuchiol 9.27\%; melatonin $-4.10 \%$; $p=$ n.s., all) nor the combination of melatonin and bakuchiol (+
$11.0 \%, p=$ n.s.) had an effect on procollagen expression (Fig. 3a). Moreover, only 3-in-1 NFS was able to increase procollagen type I levels beyond those in unirradiated skin $(+45.5 \%$, $p=$ n.s.).

\section{Effect of 3-in-1 NFS on Tropoelastin Levels}

Treatment with 3-in-1 NFS also increased expression levels of tropoelastin in the dermis of irradiated skin explants. Compared to untreated irradiated skin, tropoelastin expression was increased by $95.8 \%(p<0.05)$ (Fig. $4 a$, b). As observed for procollagen type I, the individual components of 3-in-1 NFS had no effect on tropoelastin expression (ATIP + 5.92\%; bakuchiol - 3.97\%; melatonin - 6.90\%; $p=$ n.s., all) (Fig. 4a). Tropoelastin expression in skin treated with the combination of melatonin and bakuchiol was increased with respect to untreated irradiated skin $(+35.1 \%, p<0.05)$, but not to the same extent as 3-in-1 NFS (Fig. 4a). Moreover, only 3-in-1 NFS increased dermal tropoelastin levels beyond those in unirradiated control skin $(+69.8 \%, p<0.05)$.

\section{DISCUSSION}

In clinical studies, 3-in-1 NFS, a night facial serum, comprising a direct antioxidant (ATIP), an indirect antioxidant (melatonin), and a polyphenol (bakuchiol), was shown to reduce facial wrinkles and improve the barrier properties and hydration levels of photoaged skin [21]. In this study we sought to understand the histological events responsible for this.

Skin serves a vital role in maintaining homeostasis by limiting passive water loss from the body and reducing the impact of chemical, physical, and microbial insults in its environment. These defensive functions are an inherent property of the SC [27, 28]. Thus, proper development and maintenance of the SC is paramount. By increasing FLG expression, a key molecule in SC formation [29], our results suggest that 3-in-1 NFS may help restore epidermal skin barrier integrity in photoaged skin. The clinically reduced TEWL following 3-in-1 NFS treatment is presumably a direct reflection of this. FLG also plays an important role in 
A

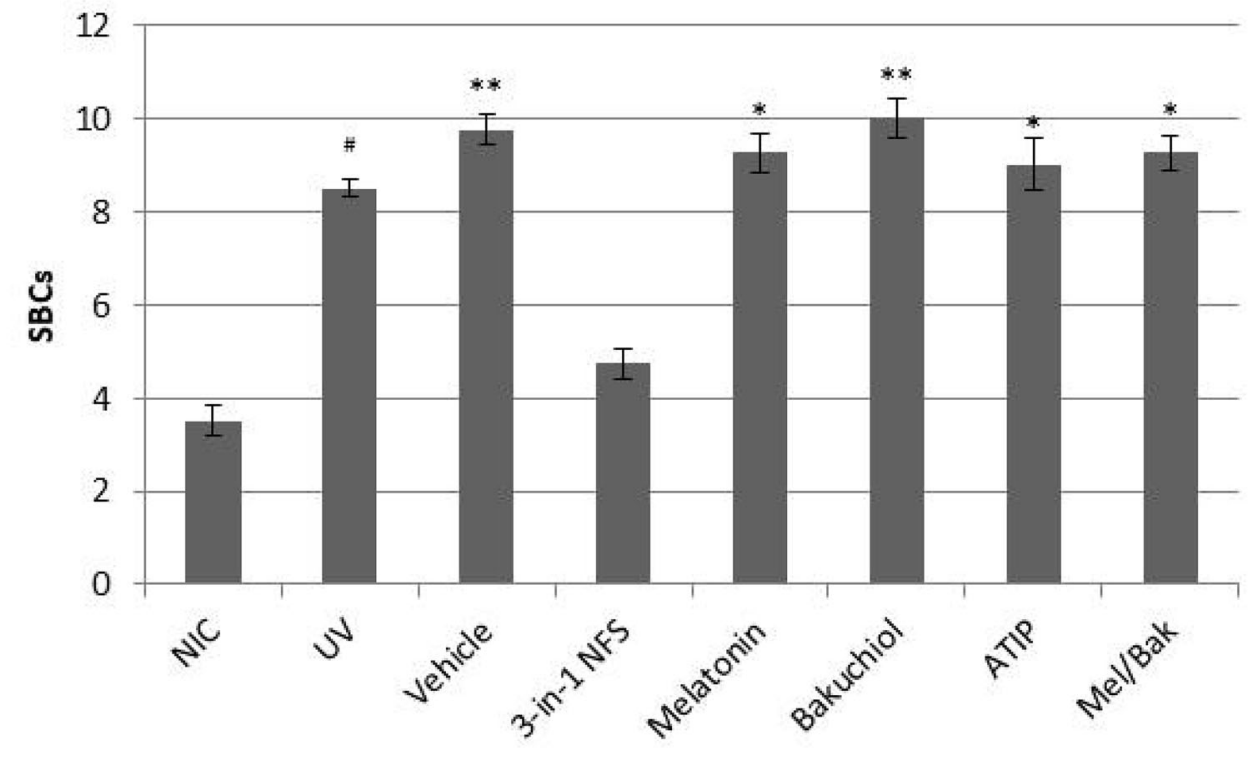

B

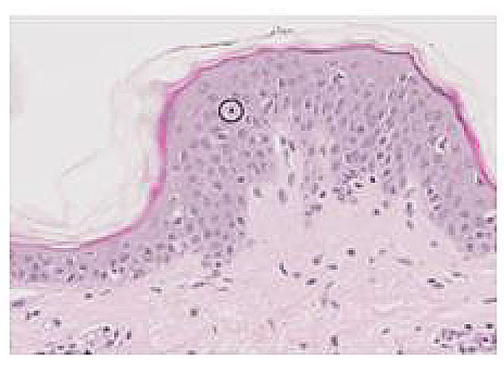

NIC

Fig. 2 Effect of 3-in-1 NFS on sunburn cell formation in UV-exposed skin explants. a Mean number of SBCs per experimental condition. Error bars correspond to the mean of 40 images. Significance levels are calculated versus untreated, unexposed control skin (NIC); ${ }^{\#} p=0.05$; ${ }^{*} p<0.05 ;{ }^{* *} p<0.01$. NIC non-irradiated control, UV

maintaining proper epidermal hydration levels following its proteolysis to produce natural moisturizing factors such as urocanic acid (UCA) and pyrrolidone carboxylic acid [30]. The upregulation of both FLG and AQP3, an aquaglyceroporin that transports water, urea, and glycerol into the cells of the epidermis [31], observed in vitro upon 3-in-1 NFS treatment may thus account for the clinically improved hydration levels.

A hallmark event of UV exposure is the occurrence of SBCs within the epidermis $[32,33]$. SBCs are keratinocytes undergoing apoptosis as a result of irreparable damage to their DNA [34]. The fact that 3-in-1 NFS significantly reduced their formation in the epidermis of UV-exposed skin explants suggests that it limits the damaging effects of UVR. Whether this protection is a direct consequence of the improved barrier function of 3-in-1 NFS-treated skin or an indirect consequence of higher antioxidant levels [35], modulation of apoptotic signaling pathways [36], or greater expression of UVB-absorbing UCA within the SC [23] remains to be established. What is clear, however, is that this photoprotective effect is a unique 


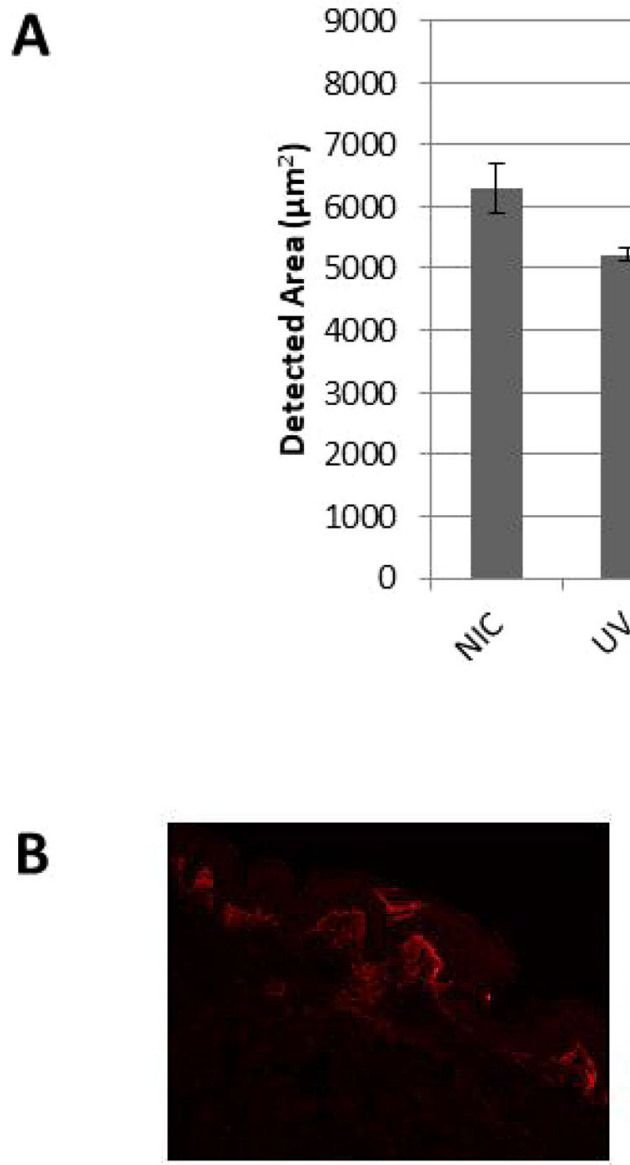

NIC

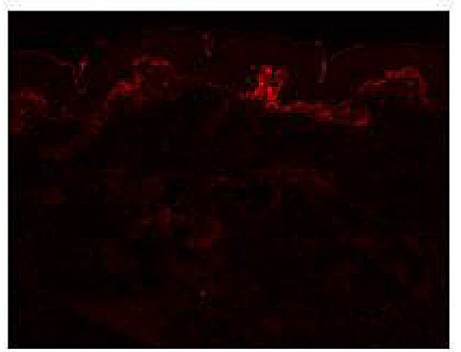

UV

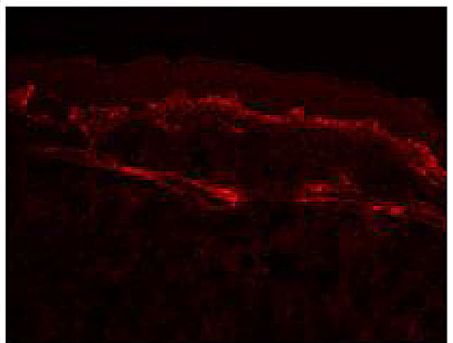

3-in-1 NFS
Fig. 3 Effect of 3-in-1 NFS on procollagen type I expression in UV-exposed skin explants. a Mean area of the dermis positive for procollagen type I expression. Error bars correspond to the mean area ( \pm SEM) of 64 images. Significance levels are calculated versus untreated UVexposed skin (UV). ${ }^{* *} p<0.01$. NIC non-irradiated

consequence of the combination of melatonin, bakuchiol, and ATIP, since none of these components individually had an effect on SBC formation. Antioxidant synergism between melatonin and vitamin $\mathrm{C}$ has previously been reported, with melatonin thought to reverse the pro-oxidant activity of vitamin $C$ [37]; however further studies are warranted to better understand the synergistic photoprotection afforded by this combination of ingredients.

After 3 months of nightly use, 3-in-1 NFS significantly reduced facial wrinkles [21]. The wrinkling and reduced elasticity typical of control, UV UV-exposed untreated skin. b Representative images showing fluorescence staining of procollagen type I (red) in skin dermis from non-irradiated skin (left), UVexposed skin (center), and 3-in-1 NFS-treated (right) skin. All images were taken at $\times 10$ magnification

photoaged skin are the result of a reduction in the amount of dermal ECM [38, 39]. Here we demonstrate that 3-in-1 NFS helps reverse this decline. Expression of both of collagen I and III was increased in HDFs, indicating that 3-in-1 NFS induces new collagen synthesis. This was confirmed in photo-irradiated skin explants, in which significant increases in precursor procollagen type I levels were observed when treated topically with 3-in-1 NFS. Additionally, we observed an increase in levels of tropoelastin, an elastin precursor, in this photoaged skin model. Notably, levels of both procollagen type I and 


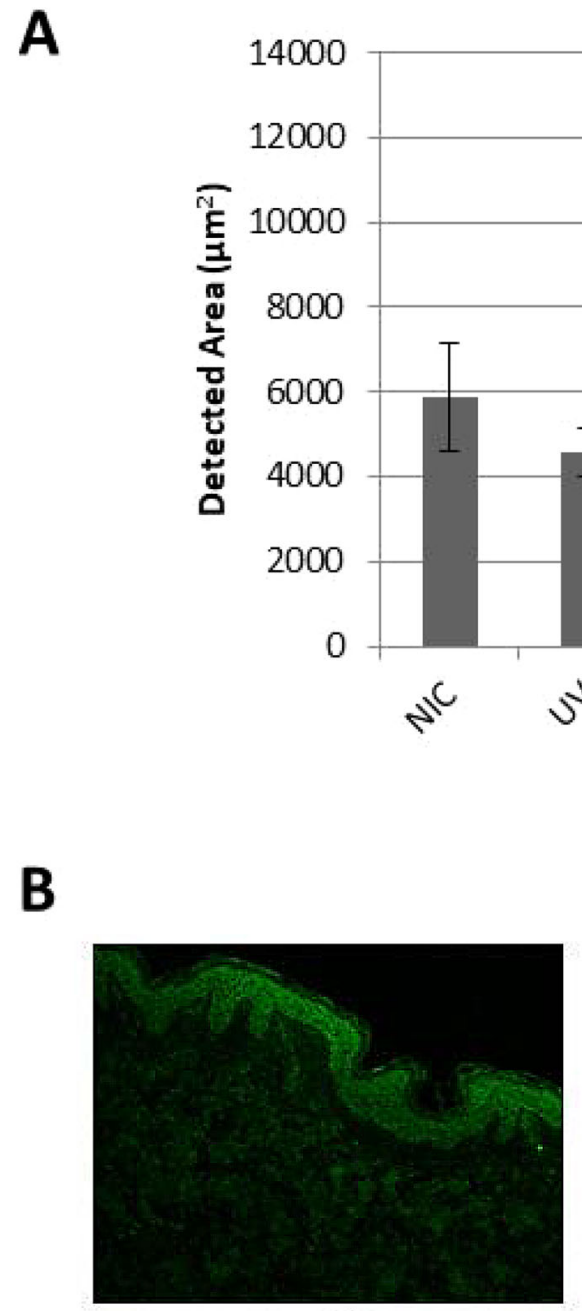

NIC

Fig. 4 Effect of 3-in-1 NFS on tropoelastin expression in UV-exposed skin explants. a Mean area of the dermis positive for tropoelastin expression. Error bars correspond to the mean area $( \pm$ SEM) in 64 images. Significance levels are calculated versus untreated UV-exposed skin (UV); ${ }^{*} p<0.05$. NIC non-irradiated control, UV UV-exposed

tropoelastin were only significantly increased in ex vivo skin explants upon 3-in-1 NFS treatment and not by its individual components, alluding again to synergism between these ingredients. Moreover, its effects on dermal proteins appeared to be limited to stimulating collagen and elastin expression, since other ECM proteins, including laminin and fibronectin, were largely unaffected by 3-in-1 NFS.

Although induction of collagen and elastin synthesis is essential for increasing dermal untreated skin. b Representative images showing fluorescence staining of tropoelastin (green) in skin dermis/ epidermis from non-irradiated skin (left), UV-exposed skin (center), and 3-in-1 NFS-treated (right) skin. All images were taken at $\times 10$ magnification

collagen and elastin levels, it is not sufficient on its own for the formation of functional collagen and elastic fibers. Hence it would therefore be necessary to examine whether functional collagen and elastin fibers are formed upon 3-in-1 NFS treatment. The fact that facial wrinkling is reduced by 3-in-1 NFS, however, suggests that it does improve the mechanical and structural integrity of the skin, alluding to the formation of functional collagen and elastin fibers. Likewise, whilst the photo-irradiated skin explant 
model used in this study effectively recapitulates a number of the principle histologic changes of photoaging, including modulating genes involved in epidermis development and ECM homeostasis (data not shown), the antiphotoaging effects of 3-in-1 NFS can only truly be gauged by determining its effects in vivo using histological samples from photoaged patients treated with 3-in-1 NFS.

\section{CONCLUSION}

Our immunocytochemical analyses of keratinocytes and fibroblasts, in combination with our histologic analysis of skin explants, suggests that some of the characteristic hallmarks of photoaging can be restored with regular use of 3-in-1 NFS. The increased expression of proteins that improve the skin's barrier function and hydration levels, and upregulation of collagen and elastin expression within the dermis, in turn accounts for the positive effects on skin appearance and function clinically. Moreover, our data suggests that the combination of melatonin, bakuchiol, and ATIP together exerts a synergistic effect on skin biology in reversing signs of aging acquired as a result of UV exposure.

\section{ACKNOWLEDGEMENTS}

We wish to thank Jessica Romero, Claudia Navarro, and Anna Rodriguez of Leitat who performed the ex vivo study described here.

Funding. This study was wholly funded by ISDIN, the manufacturer of 3-in-1 NFS. The Rapid Service Fee was funded by ISDIN.

Authorship. All authors had full access to all of the data in this study and take complete responsibility for the integrity of the data and accuracy of the data analysis. All named authors meet the International Committee of Medical Journal Editors (ICMJE) criteria for authorship for this article, take responsibility for the integrity of the work as a whole, and have given their approval for this version to be published.
Disclosures. Mridvika Narda and Corinne Granger are employees of ISDIN, the manufacturer of 3-in-1 NFS. Anthony Brown is a paid consultant to ISDIN. Béatrice Muscatelli-Groux and Jean A Grimaud are employees of Matriscience who were paid by ISDIN to perform the experimental work in this study.

Compliance with Ethics Guidelines. All human skin explants used in this study were obtained from abdominal surgical residues after written informed consent from the donors and in full respect of the Declaration of Helsinki and article L.1245 of the French Public Health Code [22]. The latter does not require any prior authorization by an ethics committee for use of surgical waste.

Data Availability. Data sharing is not applicable to this article as no datasets were generated or analyzed during the current study.

Open Access. This article is licensed under a Creative Commons Attribution-NonCommercial 4.0 International License, which permits any non-commercial use, sharing, adaptation, distribution and reproduction in any medium or format, as long as you give appropriate credit to the original author(s) and the source, provide a link to the Creative Commons licence, and indicate if changes were made. The images or other third party material in this article are included in the article's Creative Commons licence, unless indicated otherwise in a credit line to the material. If material is not included in the article's Creative Commons licence and your intended use is not permitted by statutory regulation or exceeds the permitted use, you will need to obtain permission directly from the copyright holder. To view a copy of this licence, visit http://creativecommons.org/licenses/by$\mathrm{nc} / 4.0 /$.

\section{REFERENCES}

1. Rittie L, Fisher GJ. Natural and sun-induced aging of human skin. Cold Spring Harb Perspect Med. 2015;5:a015370. 
2. Fisher GJ, Datta S, Wang Z, et al. c-Jun-dependent inhibition of cutaneous procollagen transcription following ultraviolet irradiation is reversed by alltrans retinoic acid. J Clin Invest. 2000;106:663-70.

3. Quan T, Little E, Quan H, Qin Z, Voorhees JJ, Fisher GJ. Elevated matrix metalloproteinases and collagen fragmentation in photodamaged human skin: impact of altered extracellular matrix microenvironment on dermal fibroblast function. J Invest Dermatol. 2013;133:1362-6.

4. Fligiel SEG, Varani J, Datta SC, Kang S, Fisher GJ, Voorhees JJ. Collagen degradation in aged/photodamaged skin in vivo and after exposure to matrix metalloproteinase-1 in vitro. J Invest Dermatol. 2003;120:842-8.

5. Uitto J. The role of elastin and collagen In cutaneous aging: intrinsic aging versus photoexposure. J Drugs Dermatol. 2008;7:s12-6.

6. Chen Z, Seo JY, Kim YK, et al. Heat modulation of tropoelastin, fibrillin-1, and matrix metalloproteinase-12 in human skin in vivo. J Invest Dermatol. $2005 ; 124: 70-8$.

7. Kligman AM, Zheng P, Lavker RM. The anatomy and pathogenesis of wrinkles. $\mathrm{Br} \mathrm{J}$ Dermatol. 1985;113:37-42.

8. Kaya G, Saurat J-H. Dermatoporosis: a chronic cutaneous insufficiency/fragility syndrome. Clinicopathological features, mechanisms, prevention and potential treatments. Dermatology. 2007;215: 284-94.

9. Pearse AD, Gaskell SA, Marks R. Epidermal changes in human skin following irradiation with either UVB or UVA. J Invest Dermatol. 1987;88:83-7.

10. Kambayashi H, Odake Y, Takada K, Funasaka Y, Ichihashi M. Involvement of changes in stratum corneum keratin in wrinkle formation by chronic ultraviolet irradiation in hairless mice. Exp Dermatol. 2003;12(Suppl 2):22-7.

11. Meguro S, Arai Y, Masukawa K, Uie K, Tokimitsu I. Stratum corneum lipid abnormalities in UVB-irradiated skin. Photochem Photobiol. 1999;69: 317-21.

12. Mukherjee S, Date A, Patravale V, Korting HC, Roeder A, Weindl G. Retinoids in the treatment of skin aging: an overview of clinical efficacy and safety. Clin Interv Aging. 2006;1:327-48.

13. Fischer TW, Kleszczynski K, Hardkop LH, Kruse N, Zillikens D. Melatonin enhances antioxidative enzyme gene expression (CAT, GPx, SOD), prevents their UVR-induced depletion, and protects against the formation of DNA damage (8-hydroxy-2'- deoxyguanosine) in ex vivo human skin. J Pineal Res. 2013;54:303-12.

14. Tan D, Reiter RJ, Manchester LC, et al. Chemical and physical properties and potential mechanisms: melatonin as a broad spectrum antioxidant and free radical scavenger. Curr Top Med Chem. 2002;2: 181-97.

15. Chaudhuri RK, Bojanowski K. Bakuchiol: a retinollike functional compound revealed by gene expression profiling and clinically proven to have anti-aging effects. Int J Cosmet Sci. 2014;36:221-30.

16. Shoji M, Arakaki Y, Esumi T, et al. Bakuchiol is a phenolic isoprenoid with novel enantiomer-selective anti-influenza a virus activity involving Nrf2 activation. J Biol Chem. 2015;290:28001-17.

17. Adhikari S, Joshi R, Patro BS, et al. Antioxidant activity of bakuchiol: experimental evidences and theoretical treatments on the possible involvement of the terpenoid chain. Chem Res Toxicol. 2003;16: 1062-9.

18. Dhaliwal S, Rybak I, Ellis SR, et al. Prospective, randomized, double-blind assessment of topical bakuchiol and retinol for facial photoageing. Br J Dermatol. 2019;180:289-96.

19. Ochiai Y, Kaburagi S, Obayashi K, et al. A new lipophilic pro-vitamin C, tetra-isopalmitoyl ascorbic acid (VC-IP), prevents UV-induced skin pigmentation through its anti-oxidative properties. J Dermatol Sci. 2006;44:37-44.

20. Maia Campos PMBG, Gianeti MD, Camargo FBJ, Gaspar LR. Application of tetra-isopalmitoyl ascorbic acid in cosmetic formulations: stability studies and in vivo efficacy. Eur J Pharm Biopharm. 2012;82:580-6.

21. Goldberg DJ, Robinson DM, Granger C. Clinical evidence of the efficacy and safety of a new 3-in-1 anti-aging topical night serum-in-oil containing melatonin, bakuchiol, and ascorbyl tetraisopalmitate: 103 females treated from 28 to 84 days. J Cosmet Dermatol. 2019;18:806-14.

22. Code de la santé publique. Version consolidée au 29 novembre 2019. https://www.legifrance.gouv.fr/ affichCode.do?cidTexte=LEGITEXT000006072665.

23. Mildner M, Jin J, Eckhart L, et al. Knockdown of filaggrin impairs diffusion barrier function and increases UV sensitivity in a human skin model. J Invest Dermatol. 2010;130:2286-94.

24. Sougrat R, Morand M, Gondran C, et al. Functional expression of AQP3 in human skin epidermis and reconstructed epidermis. J Invest Dermatol. 2002;118:678-85. 
25. Woodley DT, Stanley JR, Reese MJ, O'Keefe EJ. Human dermal fibroblasts synthesize laminin. J Invest Dermatol. 1988;90:679-83.

26. Knott A, Drenckhan A, Reuschlein $\mathrm{K}$, et al. Decreased fibroblast contractile activity and reduced fibronectin expression are involved in skin photoaging. J Dermatol Sci. 2010;58:75-7.

27. Elias PM, Choi EH. Interactions among stratum corneum defensive functions. Exp Dermatol. $2005 ; 14: 719-26$.

28. Elias PM. Stratum corneum defensive functions: an integrated view. J Invest Dermatol. 2005;125: 183-200.

29. McGrath JA, Uitto J. The filaggrin story: novel insights into skin-barrier function and disease. Trends Mol Med. 2008;14:20-7.

30. Boer M, Duchnik E, Maleszka R, Marchlewicz M. Structural and biophysical characteristics of human skin in maintaining proper epidermal barrier function. Postepy Dermatol Alergol. 2016;33:1-5.

31. Chrispeels MJ, Agre P. Aquaporins: water channel proteins of plant and animal cells. Trends Biochem Sci. 1994;19:421-5.

32. Danno K, Horio T. Sunburn cell: factors involved in its formation. Photochem Photobiol. 1987;45: 683-90.
33. Young AR. The sunburn cell. Photodermatol. Denmark. 1987;4:127-34.

34. Van Laethem A, Claerhout S, Garmyn M, Agostinis P. The sunburn cell: regulation of death and survival of the keratinocyte. Int J Biochem Cell Biol. 2005;37:1547-53.

35. Miyachi Y, Horio T, Imamura S. Sunburn cell formation is prevented by scavenging oxygen Intermediates. Clin Exp Dermatol. 1983;8:305-10.

36. Moriyama M, Moriyama H, Uda J, Matsuyama A, Osawa M, Hayakawa T. BNIP3 plays crucial roles in the differentiation and maintenance of epidermal keratinocytes. J Invest Dermatol. 2014;134: 1627-35.

37. Gitto E, Tan DX, Reiter RJ, et al. Individual and synergistic antioxidative actions of melatonin: studies with vitamin $\mathrm{E}$, vitamin $\mathrm{C}$, glutathione and desferrioxamine (desferoxamine) in rat liver homogenates. J Pharm Pharmacol. 2001;53: 1393-401.

38. Montagna W, Carlisle K. Structural changes in ageing skin. Br J Dermatol. 1990;122:61-70.

39. Bernstein EF, Uitto J. The effect of photodamage on dermal extracellular matrix. Clin Dermatol. 1996;14:143-51. 